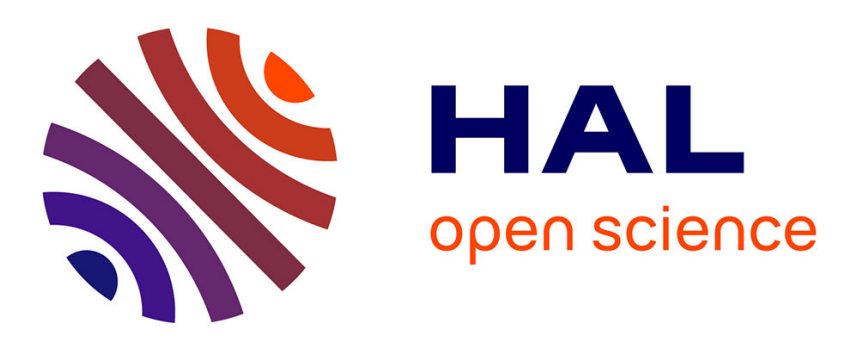

\title{
Enrichissement automatique de données par analyse spatiale pour la généralisation de réseaux
}

\author{
Guillaume Touya
}

\section{To cite this version:}

Guillaume Touya. Enrichissement automatique de données par analyse spatiale pour la généralisation de réseaux. SAGEO 2008, Jun 2008, Montpellier, France. hal-02408591

\section{HAL Id: hal-02408591 \\ https://hal.science/hal-02408591}

Submitted on 13 Dec 2019

HAL is a multi-disciplinary open access archive for the deposit and dissemination of scientific research documents, whether they are published or not. The documents may come from teaching and research institutions in France or abroad, or from public or private research centers.
L'archive ouverte pluridisciplinaire HAL, est destinée au dépôt et à la diffusion de documents scientifiques de niveau recherche, publiés ou non, émanant des établissements d'enseignement et de recherche français ou étrangers, des laboratoires publics ou privés. 


\title{
Enrichissement automatique de données par analyse spatiale pour la généralisation de réseaux
}

\author{
Guillaume Touya \\ Laboratoire COGIT, Institut Géographique National \\ 2 Avenue Pasteur 94160 Saint-Mandé Cedex \\ \{prénom.nom\}@ign.fr
}

RÉSUMÉ. La généralisation est un processus qui vise à réduire le niveau de détail d'une base de données géographiques dans le but de satisfaire de nouvelles spécifications. La sélection est une étape importante de ce processus qui consiste à choisir en fonction du contexte géographique et des besoins les objets qui devraient apparaître dans la base de données généralisée. Cet article présente une méthode générale pour la sélection automatique de réseaux géographiques basée sur l'enrichissement de données par analyse spatiale. La base de données géographiques initiale est enrichie par la reconnaissance d'informations, de structures qui n'étaient pas explicites. Par exemple, dans un réseau routier, une aire d'autoroute est un ensemble de tronçons de route connectés entrant et sortant de l'autoroute. Les processus de sélection, plus spécifiques à chaque thème, prennent en compte cet enrichissement et traitent de manière adaptée ces structures qui sont essentielles pour les futures applications de la base de données généralisée (analyse, cartographie). La méthode présentée est mise en oeuvre pour les réseaux routier et hydrographique puis testée sur des données topographiques issues des bases de données de l'Institut Géographique National. L'intérêt d'un tel enrichissement pour d'autres objectifs que la généralisation est étudié.

ABSTRACT. Generalisation is a process that seeks to reduce the level of detail of a geographic database in order to meet new specifications. Selection is a major step of the process that consist in choosing the objects that will be part of the generalised database according to geographical context. This paper presents a general method for automated selection of geographical networks based on data enrichment by spatial analysis. The initial database is enriched with implicit information or structure recognition. For instance, in a road network, highway services are groups of connected roads with enter and exit to the highway. The selection process, specific for the different themes, takes the enriched data into account and handle such structures that are essential for the future applications of the database (analysis, cartography) with appropriate treatment. The presented method is carried out on road and river networks and applied to topographic data coming from IGN databases. The interest of such enrichments for other application is also considered.

MOTS-CLÉS : généralisation, sélection, réseaux, analyse spatiale, graphes.

KEYWORDS: generalisation, selection, network, spatial analysis, graphs. 


\section{Introduction}

La généralisation est un processus qui vise à réduire le niveau de détail d'une base de données géographiques dans le but de satisfaire de nouvelles spécifications. Lorsque l'objectif est de produire une carte à une certaine échelle, on parle de généralisation cartographique. Lorsque l'objectif est la dérivation d'une nouvelle base de données géographiques, on parle de généralisation de modèle ou de base de données. La généralisation de base de données peut aussi être un préalable à une généralisation cartographique. La sélection est une étape clé du processus de généralisation de base de données : elle consiste à choisir parmi les objets géographiques de la base de données initiale ceux qui feront partie de la base de données généralisée car répondant aux spécifications de cette dernière. Pour savoir si un objet répond aux spécifications, il est souvent nécessaire de faire appel au contexte de l'objet et à des concepts géographiques implicites, ce qui rend ce processus complexe. Cet article présente une méthode générale pour la sélection des réseaux géographiques basée sur l'enrichissement de données par analyse spatiale. Cette méthode est mise en oeuvre pour la sélection des réseaux routiers et hydrographiques.

L'enrichissement de données consiste à détecter et expliciter dans une base de données des informations, des concepts ou des structures géographiques implicites. Par exemple, sans que l'information soit explicite dans les données, un humain peut, en visualisant un réseau routier, reconnaître une aire d'autoroute (Figure 1) : c'est un ensemble de tronçons de route connectés entre eux dont l'un permet de sortir de l'autoroute et un autre d'y rentrer. Dans ce cas particulier, en se basant sur nos connaissances géographiques, l'enrichissement consiste à détecter automatiquement ce groupe de tronçons, à le qualifier et à l'intégrer à la base de données.

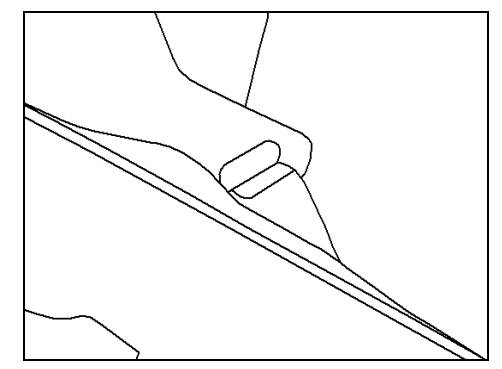

Figure 1. Une aire d'autoroute dans un réseau routier : un ensemble de tronçons dont la structure fait sens.

L'enrichissement de données par analyse spatiale est jugé comme primordial pour la généralisation par de nombreux auteurs (McMaster et Shea, 1988, Weibel et Dutton, 1998, Mackaness et Edwards, 2002). L'enrichissement dans les réseaux a 
d'ailleurs été étudié, particulièrement pour les routes (Heinzle et Anders, 2007). Ces travaux font apparaître que les réseaux géographiques contiennent des structures importantes pour la compréhension de ces données géographiques telle qu'un échangeur routier ou un boulevard périphérique dans un réseau routier ou un delta dans un réseau hydrographique. Différents travaux de recherches traitent de la sélection du réseau routier (Richardson et Thomson, 1996, Thomson et Richardson 1999) et du réseau hydrographique (Horton, 1945, Thomson et Brooks, 2000) mais ne prennent pas en compte toutes ces informations et structures implicites.

L'objectif de ce travail est d'une part d'avoir des méthodes d'analyse spatiale pour expliciter le contexte spatial dans les réseaux et détecter les structures caractéristiques de ces réseaux. Pour cela, nous allons adapter des méthodes issues de la littérature et proposer de nouvelles méthodes d'analyse spatiale. D'autre part, l'objectif est de concevoir une méthode générale pour la sélection des réseaux se basant sur cet enrichissement par analyse spatiale pour mieux traiter le contexte géographique des réseaux et pour maintenir durant le processus les structures importantes des réseaux. Le but est également de mettre en oeuvre la méthode pour des processus de sélection des réseaux routiers et hydrographiques.

La deuxième partie de cet article présente la méthode générale proposée de sélection des réseaux. La troisième décrit les enrichissements proposés selon deux axes : faciliter le processus de sélection et maintenir les structures caractéristiques. La quatrième partie présente les processus de sélection mis en oeuvres pour les réseaux routiers et hydrographiques avec une application sur des données topographiques issues des bases de données de l'Institut Géographique National (IGN). La cinquième partie propose des idées pour utiliser les enrichissements à d'autres fins que la généralisation. Enfin, la dernière partie dresse le bilan de ce travail et expose quelques perspectives de recherche.

\section{Méthodologie proposée pour la sélection des réseaux}

Afin de bien comprendre et de résoudre au mieux tous les problèmes posés par la sélection de réseau lorsque l'on souhaite tenir compte des structures caractéristiques, nous avons implémenté plusieurs méthodes issues de la littérature et effectué des tests avec ces méthodes. La Figure 2 montre la méthode générale que nous proposons pour résoudre ces problèmes.

La première étape est donc l'enrichissement de la base de données par les informations et structures implicites nécessaires pour répondre aux spécifications de la base de données généralisée. Nous appelons structure tout concept géographique rendu explicite par l'enrichissement. Nous appelons structure caractéristique une structure qui nécessite un traitement particulier pour maintenir sa compréhension après sélection. Un échangeur routier est un exemple de structure caractéristique du réseau routier. Les données enrichies servent d'entrée au processus de sélection mais également de contrôle car certains des enrichissements guident le processus en 
explicitant le contexte géographique des différents objets. Par exemple, la présence de méandres sur une rivière va guider la sélection de manière à préserver ces méandres.

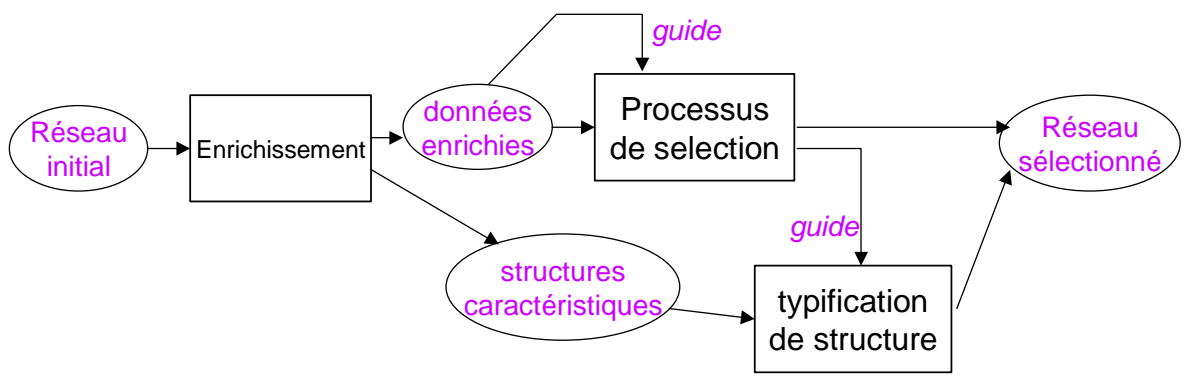

Figure 2. Schéma de la méthode générale de sélection des réseaux basée sur l'enrichissement de données dans un formalisme de type SADT.

L'étape suivante est alors le processus de sélection qui, guidé par les enrichissements, va travailler sur les données enrichies pour choisir celles qui feront partie du réseau sélectionné. Cette partie est plus spécifique à chaque réseau : par exemple, pour le réseau routier, la sélection est décomposée en trois étapes, la sélection en zone rurale, la sélection en zone urbaine et la vérification de la continuité à l'interface des deux zones. Enfin, la dernière étape de la méthode générale est la typification des structures caractéristiques du réseau. Il s'agit de diminuer le niveau de détail de la structure tout en maintenant ou en caricaturant ses caractéristiques principales. Cette étape est principalement effectuée par des algorithmes ad-hoc qui sont guidés par les résultats de la sélection sur le réseau général. Par exemple, dans le cas d'une zone d'irrigation dans un réseau hydrographique, identifiable par des tronçons formant un quadrillage, la typification consiste en une réduction du nombre de maille tout en conservant la forme d'un quadrillage. Ces trois différentes étapes sont détaillées dans les parties suivantes à travers leur mise en oeuvre pour la sélection des réseaux routiers et hydrographiques.

\section{Enrichissement de données par analyse spatiale}

Dans le cadre de la méthode de sélection que nous proposons, l'enrichissement de données par analyse spatiale a deux objectifs principaux : faciliter et guider le processus de sélection d'une part et expliciter des structures caractéristiques du réseau d'autre part qui pourront être maintenues par notre méthode générale. Ces objectifs sont illustrés dans les paragraphes suivants par des exemples de mise en oeuvre pour les réseaux routier et hydrographique. 
Enrichissement de données 5

\subsection{L'enrichissement pour faciliter et guider le processus de sélection}

L'enrichissement permet de reconnaître les structures, les relations ou les phénomènes géographiques et facilite donc la sélection en rendant le contexte explicite. S'il utilise ce contexte explicite, le processus de sélection est plus aisé et plus efficace car il est guidé par le contexte comme c'est le cas pour la méthode que nous proposons. Dans le cas des réseaux, l'enrichissement clé pour servir de base à la sélection est le recours au principe de bonne continuité du Gestalt (Thomson et Richardson, 1999). Les psychologues du Gestalt ont étudié les différents mécanismes cérébraux du regroupement perceptuel (Wertheimer, 1923). Le principe de bonne continuité permet de créer des groupes perceptuels appelés "strokes" (Thomson et Richardson, 1999) qui sont des ensembles de tronçons qui semblent se suivre de manière continue comme si on les avaient dessinés d'un seul trait. Ces strokes ont des vraies significations géographiques car les réseaux sont des phénomènes assez continus (Figure 3). Nous avons adapté ces travaux pour notre méthode et nous les avons enrichi dans le cas des réseaux hydrographiques où nous parlons de "cours d'eau continus" car le principe de continuité est ici contraint par des continuités attributaires comme le nom du cours d'eau. Dans notre méthode, une partie de la sélection est ensuite appliquée sur les strokes plutôt que sur les tronçons.

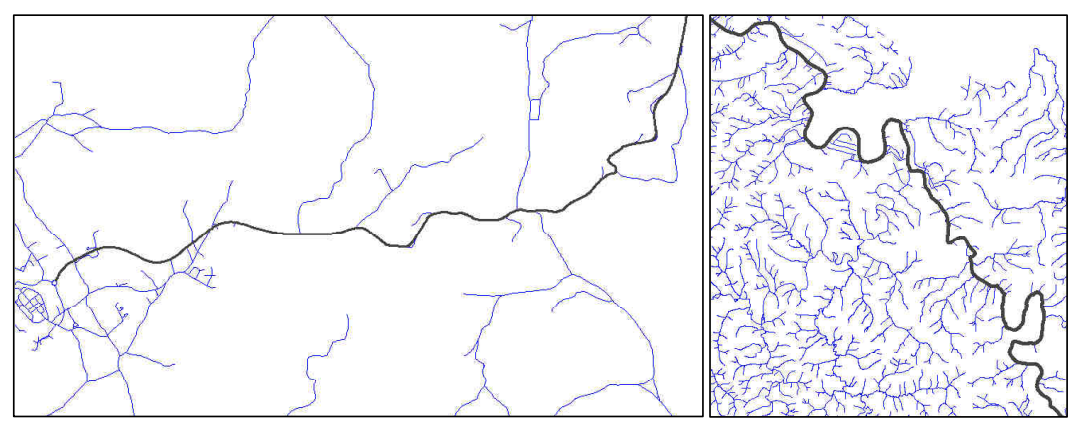

Figure 3. A gauche, un stroke de réseau routier et à droite un stroke de réseau hydrographique ou " cours d'eau continu".

L'identification des impasses dans le réseau routier est également un enrichissement important. De nombreuses spécifications de sélection distinguent en effet les impasses des autres routes. Nous avons repris la méthode de (Duchêne, 2005) qui considère le réseau routier comme un graphe et s'intéresse aux faces de ce graphe. Les tronçons se trouvant à l'intérieur d'une face sont considérés comme des impasses. Cette méthode permet de reconnaître des impasses complexes (Figure 4). Un autre enrichissement non directement lié au réseau routier sert de base au processus de sélection : la délimitation de l'étendue des villes permet de distinguer la sélection des rues en ville de la sélection des routes en zone rurale (Figure 4). Nous avons utilisé parmi les différentes méthodes existantes celle de (Boffet, 2001). 


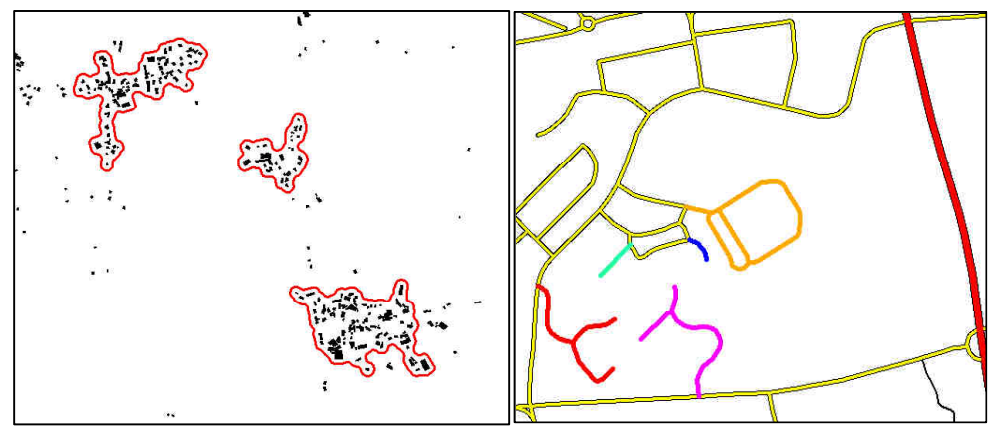

Figure 4. A gauche, création des objets villes à partir de (Boffet, 2001) et à droite des configurations d'impasses complexes détectées (Duchêne, 2005).

D'autres enrichissements permettent de guider le processus de sélection (Figure 2). Dans le cas des réseaux routiers, la classification des carrefours simples (croisements de tronçons routiers) et des carrefours complexes (configuration particulières de carrefours simples) permet de mieux contrôler la sélection. Notre classification des carrefours simples s'inspire de (Grosso, 2004) et distingue les carrefours en fourche, en $\mathrm{Y}$, en $\mathrm{T}$ ou en croix (Figure 5). Afin de caractériser les carrefours complexes, nous avons repris la méthode de (Sheeren, 2002) pour les ronds-points et développé de nouvelles méthode pour les pattes d'oies, les voies à chaussées séparées ou les carrefours décalés (Figure 5).

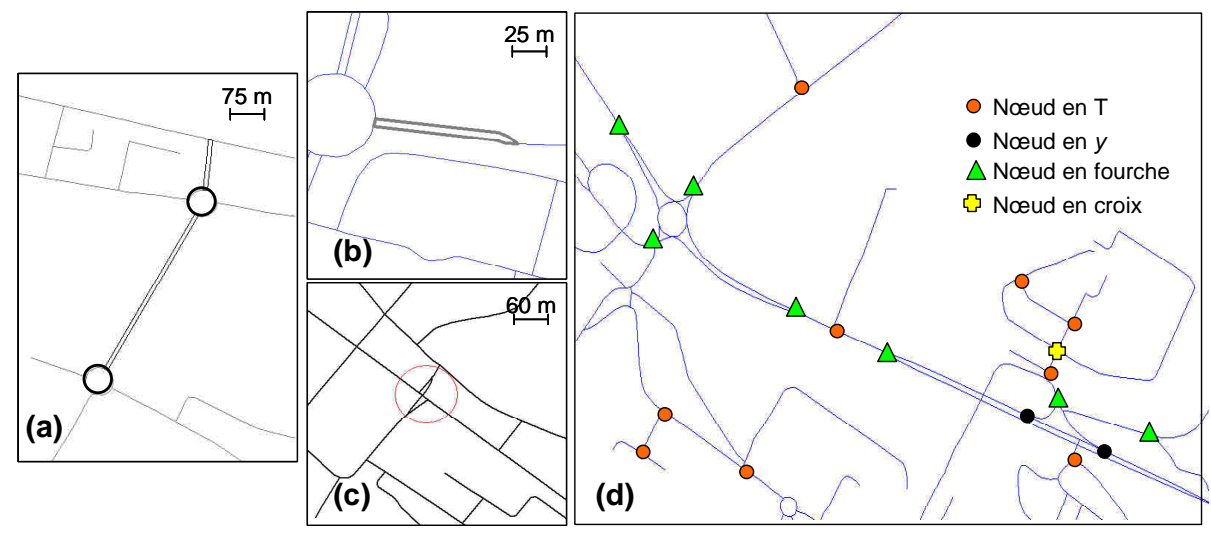

Figure 5. Classification automatique de carrefours simples et complexes: (a) rondspoints et voies chaussées séparées (b) patte d'oie (c) carrefour décalé (d) carrefours simples (Touya, 2007a). 
Enrichissement de données 7

\subsection{Méthodes de détection des structures caractéristiques}

$\mathrm{Du}$ fait de leur origine, naturelle pour les réseaux hydrographiques ou anthropique pour les réseaux routiers, ferrés ou parfois hydrographiques, des structures, des motifs apparaissent dans les réseaux. Ils sont même structurants dans l'espace géographique au point de devoir être maintenus durant la sélection. Chacune de ces structures caractéristique possède ses spécificités et leur détection par analyse spatiale requiert un algorithme adapté. Nous ne traitons dans cette partie que quatre structures caractéristiques dont nous avons conçu les méthodes de détection.

Les réseaux routiers sont parfois utilisés pour des applications de navigation pour lesquelles la détection des aires d'autoroute est nécessaire. Elles se caractérisent par un ensemble de tronçons de route connectés entre eux dont l'un permet de sortir de l'autoroute et un autre d'y rentrer. Nous les détectons en deux temps : nous repérons d'abord les entrées et sorties d'autoroutes à partir des nœuds en Y connectés à une autoroute et à partir de l'orientation du carrefour par rapport à cette autoroute (Figure $6 a)$; nous identifions ensuite les petites faces du graphe routier situées d'un côté de l'autoroute entre une sortie et une entrée. Nous prenons alors l'enveloppe convexe de l'ensemble des tronçons de ces faces pour obtenir l'étendue de l'aire d'autoroute (Figure 6b).

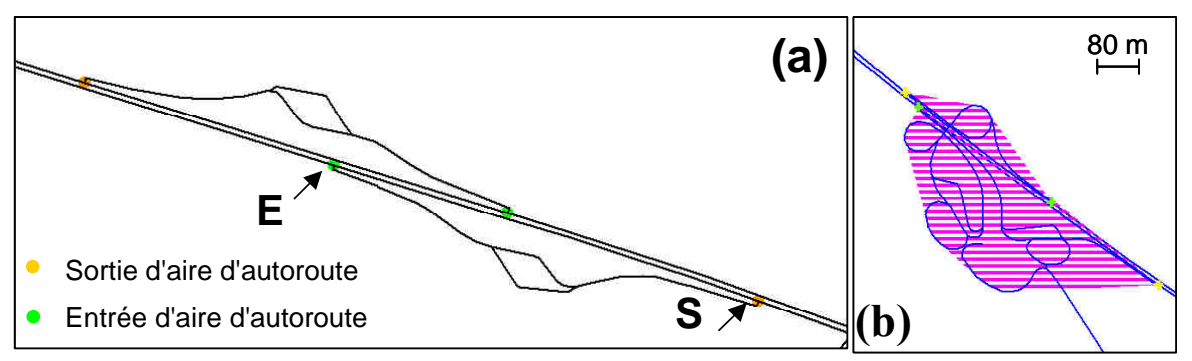

Figure 6. (a) identification automatique des entrées et sorties d'aires d'autoroute. (b) détection de la structure formée de l'ensemble de tronçons routiers.

Les échangeurs autoroutiers sont une autre structure caractéristique des réseaux routiers. D'après (Grosso, 2004, Heinzle et Anders, 2007), ils se caractérisent par une forte densité de nœuds en fourche et en Y (Figure 7). Nous exploitons cette remarque en formant donc des groupes de nœuds par agglomération de proche en proche en utilisant la distance curviligne par le réseau. Ces groupes sont ensuite discriminés suivant la présence de bâtiments à l'intérieur (il n'y a pas de bâtiment au milieu d'un échangeur). Toutes les routes incluses dans l'enveloppe convexe du groupe de nœuds plus celles connectées topologiquement au groupe composent l'échangeur routier. 


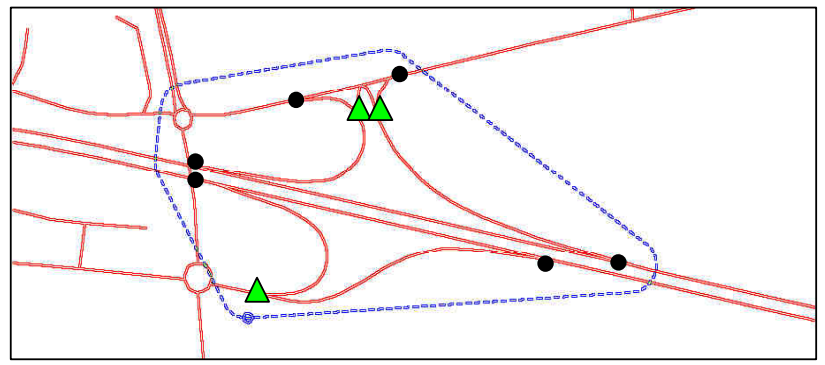

Figure 7. Un échangeur routier détecté automatiquement par agglomération de nœuds en y (ronds) et en fourche (triangles), représenté par un buffer de l'enveloppe de ces nouds.

Dans un réseau hydrographique linéaire, les différents bras d'une rivière sont souvent tous représentés dans la base de données, marquant la présence d'une ou plusieurs îles sur la rivière. Nous considérons ces îles comme une structure caractéristique des réseaux hydrographiques que nous détectons afin de les maintenir ou de les simplifier de façon pertinente en fonction des spécifications. Les îles simples sont les petites faces du graphe hydrographique. Les îles complexes sont détectées par agglomération d'îles simples adjacentes (Figure 8). Le contour est alors déterminé par voisinage topologique (les tronçons de cours d'eau extérieurs).

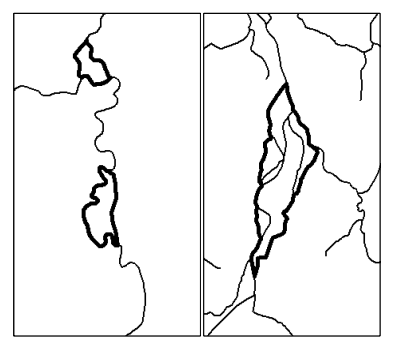

Figure 8. Des îles simples à gauche et une île complexe composée d'îles adjacentes à droite. Le contour est créé automatiquement.

Le dernier exemple de structures caractéristiques que nous présentons est la zone d'irrigation dans un réseau hydrographique. Il s'agit, dans des zones plates, de canaux artificiels et souvent tracés en quadrillage qui permettent l'irrigation des champs. Nous les détectons par agrégation : dans les zones plates, les sources et les pertes (extrémités du réseau) sont regroupées selon un critère de distance. Les groupes sont ensuite discriminés suivant leur forme (qui doit être assez compacte) et suivant la densité en petits tronçons (Figure 9a). Les tronçons inclus dans la zone d'irrigation sont ensuite discriminés en tronçons naturels (longs et sinueux) et en tronçons 
artificiels (courts et droits) car les réseaux d'irrigation s'appuient sur le réseau naturel et les deux types de tronçons coexistent dans une zone d'irrigation.

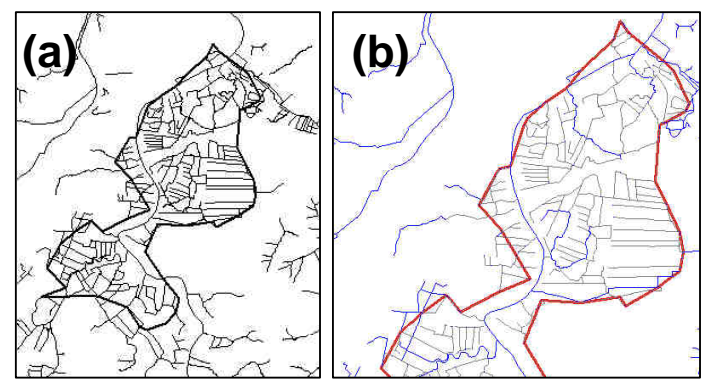

Figure 9. (a) une zone d'irrigation détectée automatiquement par notre méthode. (b) discrimination des tronçons naturels (bleu) et artificiels (gris) dans une zone d'irrigation.

Les méthodes de détection automatique des structures (dont les structures caractéristiques) ont été évaluées par des tests et les résultats sont jugés satisfaisant. Les tests ont étés effectués sur tout un département (Pyrénées-Atlantiques) issu de la BD Topo ${ }^{\circledR}$ de l'IGN. Les différentes images présentées dans cette partie sont issues de ces résultats. Pratiquement toutes les structures sont détectées. A titre d'exemple, après validation visuelle, la méthode de détection trouve bien les 269 ronds-points de la zone et pas un de plus. Mais une aire de repos sur 13 est sous-détectée car l'entrée est située exactement au niveau d'un pont (ce qui donne un nœud de degré 5 qui n'est pas choisi comme entrée). Il y a aussi parfois un peu de sur-détection. Ainsi, pour les zones d'irrigations 2 des 20 zones détectées n'en sont pas après vérifications visuelle. De plus, en travaillant sur un département entier (100 000 tronçons routiers et 50000 tronçons hydrographiques), nous pouvons assurer des temps de calcul faibles (moins d'une minute pour détecter les aires de repos).

\section{Processus de sélection}

\subsection{La sélection des routes}

Comme préconisé par (Ruas, 1999, p.163) du fait de différences fondamentales entre les réseaux routiers urbains et ruraux (forme, densité, fonction), le processus de sélection que nous proposons est séparé en trois phases : la sélection en zone rurale, la sélection en zone urbaine et la vérification de la continuité à l'interface des deux zones.

Notre sélection en zone rurale, complète l'idée de (Richardson et Thomson, 1996). Elle ainsi est basée sur l'évaluation de la fréquentation des routes par calcul de plus courts chemins entre des points d'attraction (aéroport, école, zone d'habitat 
résidentiel). Mais notre sélection s'appuie également sur les strokes qui sont qualifiés par leur longueur et leur nombre d'intersection et sur les impasses. Sont sélectionnées les routes à forte fréquentation ou appartenant à un stroke important.

La sélection en zone urbaine s'appuie sur une approche complètement différente qui poursuit les travaux de (Ruas, 1999, p.163). L'idée est d'agréger les faces du graphe routier et d'éliminer les rues séparant deux faces agrégées. L'agrégation se base sur la fréquentation et la longueur du stroke de la rue séparatrice mais aussi sur la taille et la forme de la face agrégée.

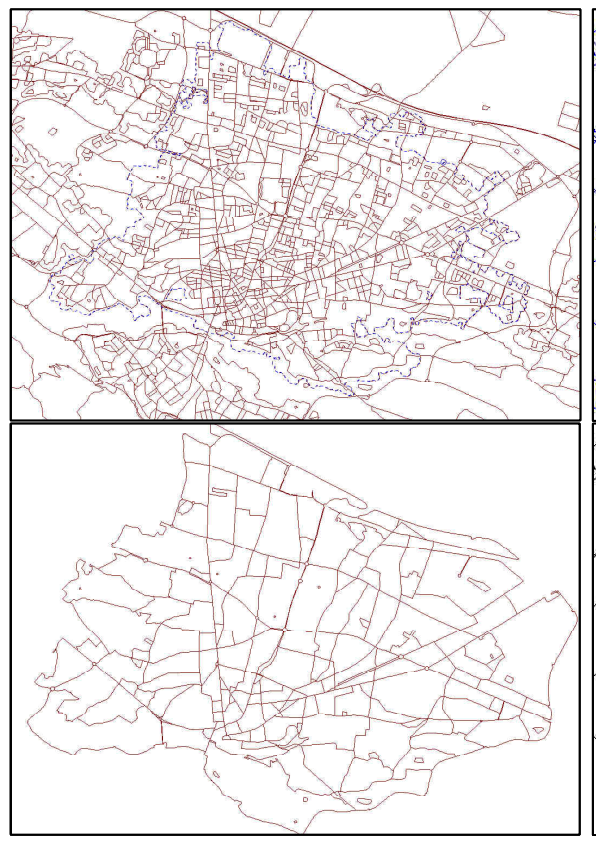

(a) Zone urbaine

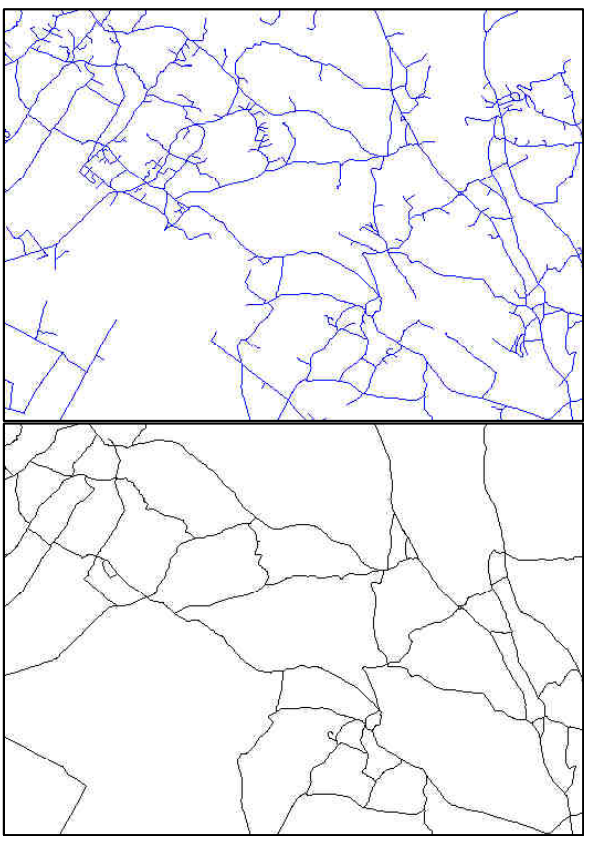

(b) Zone rurale

Figure 10. (a) Une zone urbaine et sa sélection en dessous. (b) Une zone rurale et sa sélection (Touya, 2007a).

Enfin, la continuité du réseau à l'interface des deux zones est assurée par un phase de vérification qui s'appuie sur les strokes. Parmi les strokes qui traversent deux zones, s'il y en a dont seulement une partie des tronçons a été sélectionnée par un des deux processus, on sélectionne après coup le reste du stroke pour assurer la continuité.

Cette méthode de sélection ainsi que les outils d'enrichissement ont été implémentés sur le SIG Clarity ${ }^{\mathrm{TM}}$ de la société 1Spatial. Des tests ont été effectués sur le réseau routier de la BD Topo ${ }^{\circledR}$ de l'IGN de résolution métrique avec des 
paramètres correspondant à différentes spécifications en sortie (pour des résolutions de bases de données différentes). La Figure 10 montre quelques résultats en zone rurale et urbaine. La méthode a été évaluée en réalisant une comparaison avec une autre base de données de l'IGN, la BD Carto ${ }^{\circledR}$ de résolution $10 \mathrm{~m}$. On obtient quasiment les mêmes tronçons en zone rurale $(97 \%$ d'arcs identiques dans la zone test) et une sélection équivalente en zone urbaine (les mêmes tronçons importants et peu d'écart, ici $2 \%$, en longueur totale de tronçons ). Ces résultats sont globalement satisfaisant.

\subsection{La sélection des cours d'eau}

Comme dans (Thomson et Brooks, 2000), la sélection des cours d'eau consiste à réaliser une classification de Horton (Horton, 1945) sur les cours d'eau continus (Figure 11). Un ordre d'importance est attribué à chaque cours d'eau continu en fonction du nombre d'affluents. Nous sélectionnons ensuite les cours d'eau continu dont l'ordre d'importance est supérieur à un seuil. De plus cette sélection est contrôlée par les informations issues de l'enrichissement. Ainsi, les îles, les deltas, les zones d'irrigation ou les méandres contraignent la sélection. Un exemple des contraintes que nous avons ajoutées est le suivant : si on sélectionne une partie d'un méandre, on doit sélectionner l'ensemble du méandre.

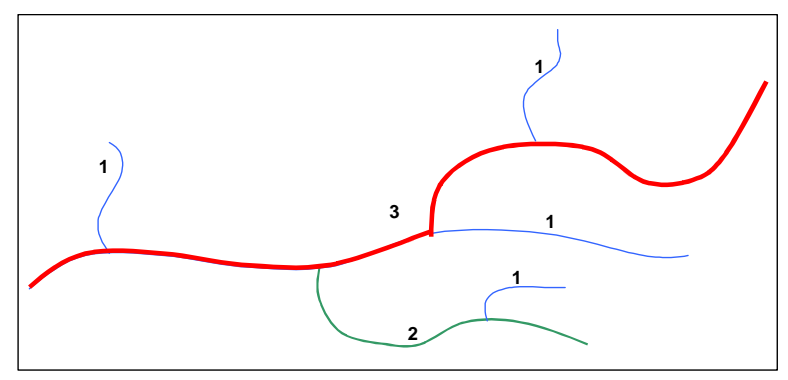

Figure 11. Classification de Horton de cours d'eau continus (créés manuellement sur ce schéma) sur laquelle s'appuie la sélection des cours d'eau.

Comme pour le réseau routier, nous avons testé notre méthode de sélection de réseau hydrographique sur le SIG Clarity ${ }^{\text {Tм }}$ et sur le réseau de la BD Topo®. La Figure 12 montre un extrait d'un des tests de sélection paramétré pour des spécifications correspondant à une base de résolution $25 \mathrm{~m}$. La méthode a été évaluée en réalisant une comparaison avec une autre base de données de l'IGN, la BD Carto ${ }^{\circledR}$ de résolution $10 \mathrm{~m}$. Dans le test utilisant comme paramètres les spécifications de cette base, on obtient un résultat équivalent ( $92 \%$ de longueur commune dont les mêmes cours d'eau principaux) mais surtout plus cohérent avec ses spécifications par endroits. 


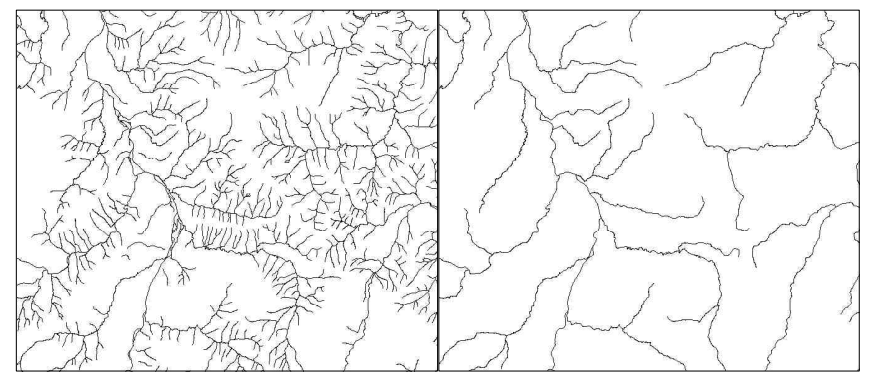

Figure 12. Résultats de la sélection sur des données BD Topo ${ }^{\circledR}$ (résolution $1 \mathrm{~m}$ ) vers des données adaptées à une base de résolution $25 \mathrm{~m}$ (Touya,2007b).

\subsection{La phase de typification}

Pour les structures caractéristiques du réseau (\$3.2), les processus de sélection généraux ne sont pas adaptés pour maintenir correctement la structure. Des méthodes de typification ad hoc sont nécessaires. Typifier consiste à "porter dans une présentation, qui en préserve la distribution reconnue, des objets de même nature qui se trouvent groupés localement par quelque processus géographique identifiable" (Hangouët, 1998, p. 227). Deux exemples sont présentés dans cette partie : les îles complexes et les échangeurs routiers.

Pour permettre plusieurs niveaux d'abstraction dans la généralisation des îles complexes, il est intéressant d'avoir recours à une agrégation hiérarchique : par exemple, les plus petites îles sont agrégées à une île voisine selon leur taille (Figure 13).

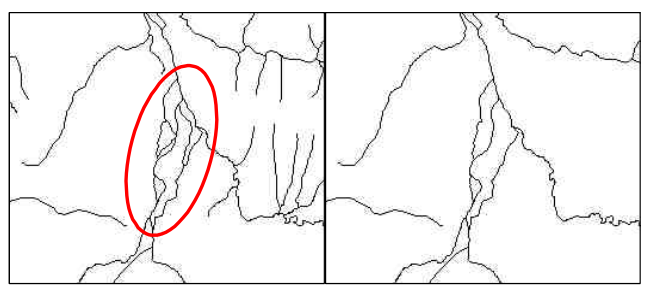

Figure 13. Une île complexe typifiée : les bras principaux sont conservés et le phénomène d'île complexe est préservé.

Pour les échangeurs routiers, nous avons adapté l'algorithme de (Mackaness et Mackechnie, 1999) qui agrège les nœuds constitutifs de l'échangeur de manière hiérarchique. Cela permet ensuite plusieurs niveaux d'élimination des nœuds dans l'échangeur (Culson, 2006) et permet de simplifier l'échangeur (Figure 14). Certains segments recréés durant la typification nécessitent une correction géométrique comme dans le cas de l'ordre 1 sur la Figure 14. 


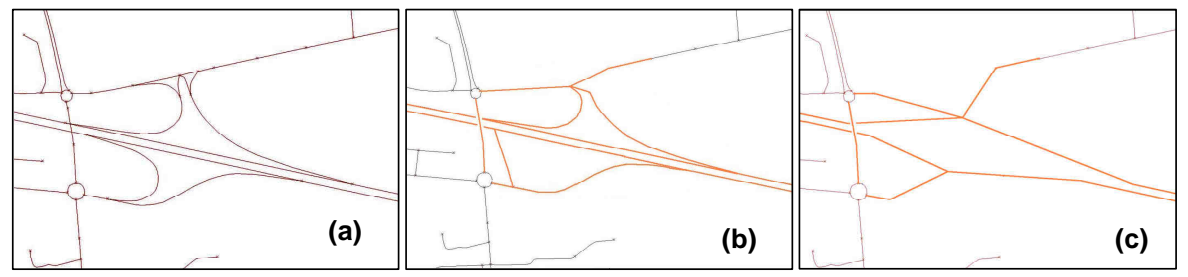

Figure 14. un échangeur routier (a) et ses formes typifiées à différents degrés (b) et (c). Des corrections géométriques sont nécessaires pour corriger les déformations.

\section{Autres applications possibles des méthodes d'enrichissement proposées}

Les enrichissements présentés dans cet article ont été conçus dans le contexte de la sélection de réseaux. Mais la plupart d'entre eux peuvent être utiles dans le cadre d'autres applications de géomatique. La partie 5.1 donne des exemples d'enrichissements développés pour la sélection pouvant servir à des analyses spatiales géographiques. La partie 5.2 décrit des exemples utiles dans le cadre de dérivations dont l'objectif n'est pas la réduction du niveau de détail.

\subsection{Application à des analyses géographiques}

Les méandres sont des éléments caractéristiques de la morphologie des rivières. Nous les avons détectés pour guider la sélection mais ils pourraient servir à des analyses géomorphologiques des réseaux hydrographiques. Nous les avons détectés en segmentant les "cours d'eau continus" (voir §3.1) en parties homogènes en sinuosité selon la méthode de (Plazanet, 1996). Les virages des parties sinueuses sont ensuite qualifiés, par des mesures classiques comme le nombre de points d'inflexion ou le rapport base/longueur, pour trouver les méandres de petite ou grande ampleur (Figure 15). Ils sont utilisés pour guider la sélection mais peuvent servir à des analyses géomorphologiques des réseaux hydrographiques.

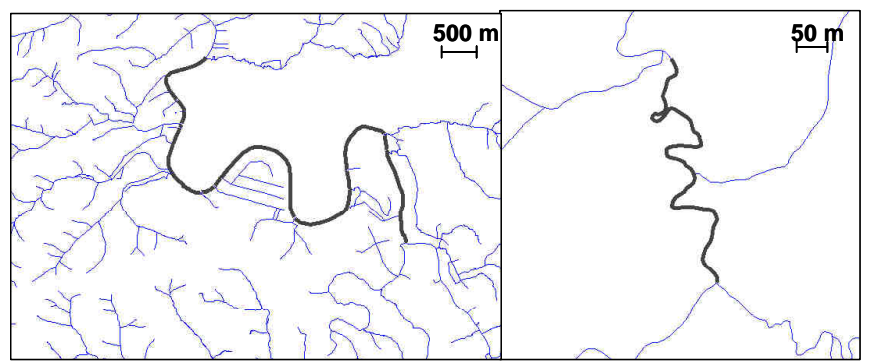

Figure 15. A gauche, des grands méandres détectés automatiquement et à droite des petits méandres. 
De même, la détection d'impasses complexes ou de structures maillées dans un réseau routier peuvent servir de support à l'analyse géographique du tissu urbain. Ainsi, certaines des méthodes d'analyse de réseaux présentées dans cet article seront utilisées dans le récent projet ANR GeOpenSim (GeOpenSim, 2008) qui vise à construire une plate-forme open source dédiée à l'analyse des tissus urbains.

\subsection{Application à des dérivations spécifiques}

La dérivation de base de données géographiques a parfois pour objectif de remplir un besoin particulier plutôt que réduire le niveau de détail. On peut par exemple vouloir obtenir une base de données routière pour faire de la navigation. Dans ce cas, il est intéressant d'enrichir les données avec des structures dont on explicite les relations avec les autres objets géographiques. La Figure 16 montre un rond-point connecté à une patte d'oie dans un réseau routier. Plusieurs relations implicites entre ces éléments et les tronçons de routes existent et leur explicitation, comme dans le schéma de données associé à la figure, peut être utile. Cela permet directement d'exprimer qu'un tronçon mène à un carrefour complexe et que trois autres tronçons permettent d'en sortir. Pour une base de données de gestion des rivières, certaines structures comme les zones d'irrigation sont des enrichissements importants.

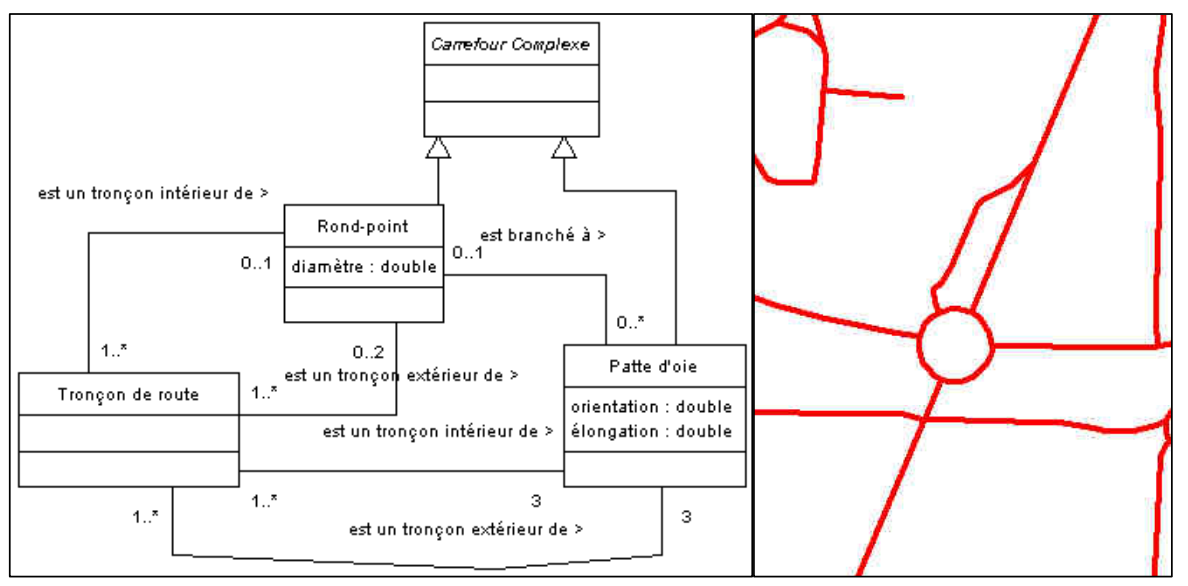

Figure 16. Schéma de données enrichi explicitant structures et relations implicites concernant la situation de l'image de droite: un rond-point et une patte d'oie.

\section{Conclusion et perspectives}

En conclusion, cet article présente une méthode générale de sélection des réseaux basé sur l'enrichissement de données par analyse spatiale. Les contribution de ce travail sont de plusieurs ordres : d'abord la sélection et l'adaptation de méthodes 
d'enrichissement issues de la littérature; le développement de méthodes d'enrichissement complémentaires; le développement de nouvelles méthodes de typification; l'adaptation de méthodes de sélection de la littérature en les guidant à l'aide des méthodes d'enrichissement; enfin, la conception de la méthode générale de sélection des réseaux basée sur l'enrichissement. La méthode a été mise en oeuvre pour la sélection des réseaux routiers et hydrographiques et testée avec succès sur des données topographiques de l'IGN. Les enrichissements développés et adaptés peuvent également être utiles pour d'autres applications que la généralisation comme des analyses géographiques ou des dérivations spécifiques.

Les perspectives sont de deux ordres. Il serait d'abord intéressant de mettre en oeuvre cette méthode sur d'autres réseaux géographiques comme les réseaux ferrés ou électriques. Par ailleurs, il serait intéressant d'approfondir la modélisation des objectifs d'une généralisation et notamment la traduction les volontés d'un utilisateur en paramètres de ces processus de sélection. L'utilisation de méthodes d'aide au paramétrage basée sur des échantillons comme (Hubert, 2008) permettrait à un utilisateur standard de spécifier ses paramètres de sélection.

\section{Bibliographie}

Boffet A., « Méthode de création d'informations multi-niveaux pour la généralisation de l'urbain ». Thèse de doctorat, Université de Marne-la-Vallée, 2001.

Culson N., « Détection et généralisation des échangeurs routiers dans une base de données cartographique vectorielle ».Mémoire de DESS, Université Paris 6. Laboratoire COGIT, IGN. 2006.

Duchêne C., « Enrichissement de données urbaines par calculs d'analyse spatiale ». Rapport interne du laboratoire COGIT, IGN. 2005.

GeOpenSim, «Les objectifs du projet GeOpenSim ». http://geopensim.ign.fr

Grosso E., «Etude des carrefours d'un réseau routier - Première approche : modélisation et implémentation d'outils de détection des carrefours (plate-forme Oxygène) ». Mémoire de DESS, Université Paris 1. Laboratoire COGIT, Institut Géographique National. 2004.

Hangouët J.-F., « Approche et méthodes pour l'automatisation de la généralisation cartographique; application en bord de ville ». Thèse de doctorat, Université de Marne-laVallée, 1998.

Heinzle F., Anders K.-H., «Characterising Space via Pattern Recognition Techniques: Identifying Patterns in Road Networks ». Mackaness W., Ruas A., Sarjakoski T. (eds) : The Generalisation of Geographic Information : Models and Applications. Elsevier (2007).

Horton, R.A., «Erosional development of Streams and their Drainage Basins:Hydrophisical approach to Quantitative Morphology ».Geo. Soc. America Bull, Vol. 56 (1945) 275-370

Hubert, F., « CartAble, système d'aide au paramétrage de traitements géographiques complexes ». Revue Internationale de Géomatique, Vol. 18, n¹, 2008. 
Mackaness W., Edwards, G., "The Importance of Modelling Pattern and Structure in Automated Map Generalisation ». In Proceedings of Joint Workshop on Multi-scale Representations of Spatial Data, Ottawa, Canada (2002).

Mackaness W., Mackechnie G.A., " Automating the Detection and Simplification of Junctions in Road Networks ». GeoInformatica, Vol. 3, Issue 2, 1999. pp. 185-200.

Plazanet C., «Enrichissement des bases de données géographiques : analyse de la géométrie des objets linéaires pour la généralisation cartographique (application aux routes) ». Mémoire de thèse 1996 (Université de Marne la Vallée).

Richardson D.E., Thomson R.C., «Integrating Thematic, Geometric and Topologic Information in the Generalization of Road Networks ». Cartographica. Volume 33 (1) 1996, pp. 75-83.

Ruas, A., « Modèle de généralisation de données géographiques à base de contraintes et d'autonomie ». Mémoire de thèse. Laboratoire COGIT (1999).

Sheeren D., « L'appariement pour la constitution de bases de données géographiques multirésolutions. Vers une interprétation des différences de représentation ». Revue Internationale de Géomatique Vol 12 n.2/2002 pp 151-168

Thomson R.C., Richardson D., «The "Good Continuation" principle of Perceptual Organization applied to the Generalization of Road Networks ", in proceedings of the $19^{\text {th }}$ ICC. ICA, Ottawa, Canada (1999).

Thomson R., Brooks R., «Efficient generalisation and abstraction of network data using perceptual grouping ». In proceedings of the $5^{\text {th }}$ GeoComputation. University of Greenwich, Kent U.K. (2000)

Touya G., «A Road Network Selection Process Based on Data Enrichment and Structure Detection ». In proceedings of $10^{\text {th }}$ ICA Workshop on Generalisation and Multiple Representation. Moscow (Russia). 2007a

Touya G., «Un processus de sélection du réseau hydrographique, basé sur la détection des structures ». Le Monde des Cartes. Revue du Comité Français de Cartographie. Num. 194. Décembre 2007b. pp. 46-56.

Weibel, R., Dutton, G., « Constraint-Based Automated Map Generalization ». In proceedings of the $8^{\text {th }}$ International Symposium on Spatial Data Handling, 1998, pp.214-224.

Wertheimer M., "Laws of organization in perceptual forms». First published as Untersuchungen zur Lehre von der Gestalt II, in Psycologische Forschung, 4, 301-350. Translation published in Ellis, W. (1938). A source book of Gestalt psychology (1923) $71-88$ 